\title{
PROPIEDADES FÍSICAS, QUÍMICAS Y ANTIOXIDANTES DE VARIEDADES DE MANGO CRECIDAS EN LA COSTA DE GUERRERO
}

\author{
PHYSICAL, CHEMICAL AND ANTIOXIDANT PROPERTIES OF MANGO VARIETIES \\ GROWN AT THE GUERRERO COAST
}

\author{
Yanik I. Maldonado-Astudillo1,2*, Heidi A. Navarrete-García', Óscar D. Ortiz-Morales ${ }^{1}$, \\ Javier Jiménez-Hernández ${ }^{1,2}$, Ricardo Salazar-López ${ }^{4}$, Irán Alia-Tejacal ${ }^{3}$ y Patricia Álvarez-Fitz ${ }^{4}$
}

\begin{abstract}
'Unidad Académica de Ciencias Químico Biológicas, Universidad Autónoma de Guerrero. Av. Lázaro Cárdenas s/n, Ciudad Universitaria sur. 39090, Col. La haciendita. Chilpancingo de los Bravo, Guerrero, México. ${ }^{2}$ Maestría en Competitividad y Sustentabilidad, Universidad Autónoma de Guerrero. Calle Pino s/n. 39640, Col. El roble, Acapulco, Guerrero, México. ${ }^{3}$ Posgrado en Ciencias Agropecuarias y Desarrollo Rural, Facultad de Ciencias Agropecuarias, Universidad Autónoma del Estado de Morelos. Av. Universidad 1001. 62210, Cuernavaca, Morelos, México. ${ }^{4}$ CoNACyT-Universidad Autónoma de Guerrero. Av. Javier Méndez Aponte No. 1, Fracc. Servidor Agrario. 39070, Chilpancingo de los Bravo, Guerrero, México.
\end{abstract}

*Autor de correspondencia (yaixma@gmail.com)

\section{RESUMEN}

El mango (Mangifera indica L.) es una fruta tropical que destaca por su particular sabor y aroma, tiene amplia aceptación y una creciente demanda en los mercados internacionales. Dado que las propiedades y composición varían con la región de cultivo, el objetivo del presente trabajo fue determinar las características físicas, químicas y actividad atrapadora de radicales libres de cuatro variedades de mango producidas en el estado de Guerrero. Se cosecharon frutos sanos en madurez fisiológica y de consumo de las variedades de mango Ataulfo, Manila, Irwin y Criollo. Las variedades sobresalientes en atributos de calidad física fueron: Ataulfo en biomasa $(387.8 \mathrm{~g})$; Irwin en firmeza $\left(37.3 \mathrm{~kg} \mathrm{~cm}^{-2}\right)$; Ataulfo Manila y Criollo en color externo (matiz $>40 \mathrm{o}$ ). La mayor acidez titulable total fue para Ataulfo ( 0.3 $\%)$; pH para Manila, Criollo e Irwin (3.5 a 3.9). No se presentaron diferencias significativas entre variedades en el contenido de sólidos solubles totales ( 8.5 a $11.3^{\circ}$ Brix), humedad (60 a $82 \%$ ) y cenizas ( 0.4 a $0.5 \%$ ). El mayor contenido de azúcares totales se presentó en la variedad Irwin (31\%) y de vitamina $C$ en Manila y Criollo (34 a $48 \mathrm{mg} \mathrm{g}^{-1}$ ). El mayor contenido de polifenoles (527.59 mg equivalentes de ácido gálico/100 g) y flavonoides (438.69 mg equivalentes de catequina/ $100 \mathrm{~g}$ ) fue para Ataulfo en frutos en madurez fisiológica y la mayor actividad atrapadora de radicales libres para extractos metanólicos de las variedades Ataulfo y Criollo. Se concluye que las variedades Ataulfo, Manila, Criollo e Irwin satisfacen los requerimientos de la Norma NMX-FF-058-SCFI-2006 de calidad de mango fresco para comercio nacional y que la variedad Ataulfo cumple con la categoría extra grande de la NOM-188-SCFI-2012. La gran actividad atrapadora de radicales libres de las variedades Manila, Irwin y Criollo les confieren valor a sus frutos como alimentos funcionales.

Palabras clave: Mangifera indica, características fisicoquímicas, Ataulfo, Manila, Irwin, Criollo.

\section{SUMMARY}

Mango (Mangifera indica L.) is a tropical fruit that stands out for its particular flavor and aroma, it is widely accepted, and it has growing demand in international markets. Since properties and composition vary according to the region of production, the aim of this study was to determine physical and chemical characteristics, as well as the free radical scavenging activity of four mango varieties harvested in the state of Guerrero. Healthy fruits of Ataulfo, Manila, Criollo and Irwin varieties were harvested at both physiological and consumption maturity. Outstanding varieties in regard to physical quality traits were Ataulfo with the highest weight $(387.8 \mathrm{~g})$; Irwin with the highest firmness $\left(37.3 \mathrm{~kg} \mathrm{~cm}^{-2}\right)$; and Ataulfo, Manila and Criollo with external color
( $>40^{\circ} \mathrm{h}$ ). Regarding chemical properties, the highest titratable acidity was found in Ataulfo (0.3\%), while the lowest $\mathrm{pH}$ was found in Manila, Criollo and Irwin (3.5 to 3.9). There were no significant differences among varieties for total soluble solids ( 8.5 to $11.3^{\circ} \mathrm{Brix}$ ), moisture (60 to $82 \%$ ) and ash content (0.4 to $0.5 \%)$. The highest content of total sugar was recorded in the Irwin variety (31\%), and the highest content of Vitamin C in Manila and Criollo (34 to $48 \mathrm{mg} \mathrm{g}^{-1}$ ). The highest content of polyphenols (527.59 mg equivalents of gallic acid/100 g) and flavonoids (438.69 mg equivalents of catechin/100 g) was observed in Ataulfo at physiological maturity, and the highest free radical scavenging activity for methanolic extract was present in Ataulfo and Criollo varieties. It is concluded that Ataulfo, Manila, Criollo and Irwin varieties meet the quality requirements of NMX-FF-058-SCFI-2006 norm for fresh mango for domestic trade, whereas the Ataulfo variety meets the extra-large category of NOM-188-SCFI-2012. The high free radical scavenging activity of the Manila, Irwin and Criollo varieties confers value to their fruits as functional food.

Index words: Mangifera indica, physical-chemical characteristics, Ataulfo, Manila, Irwin, Criollo.

\section{INTRODUCCIÓN}

El género Mangifera comprende alrededor de 50 especies nativas del sureste de Asia. El mango (Mangifera indica L.) se originó en la región Indo-Birmana y es una de las frutas tropicales más importantes del mundo por su producción, superficie cultivada, y popularidad; su producción global es superior a 27 millones de toneladas, lo que lo ubica como el mayor cultivo tropical (FAOSTAT, 2012). México ha sido el principal país oferente de mango en los mercados internacionales y Estados Unidos es su principal comprador, ya que recibe el $56 \%$ del total de las exportaciones mexicanas (Huang y Huang, 2007).

Esta destacada participación de México en el mercado mundial del mango se debe a que cuenta con las condiciones climáticas adecuadas para el desarrollo de este cultivo. México ocupa el sexto lugar en producción a nivel mundial y el estado de Guerrero es el líder nacional con una participación de $22 \%$ del volumen total, seguido de Nayarit (17 $\%)$ y Sinaloa (14\%); estos tres estados en conjunto aportan 
el $53 \%$ de la producción nacional (SAGARPA, 2014). Las principales variedades cultivadas en Guerrero son Ataulfo, Manila, Haden, Irwin, Kent, Keitt y Criollo; las tres primeras son las que se comercializan en el mercado internacional (CONASPROMANGO, 2012).

En México se han impulsado campañas de difusión del valor gastronómico y nutricional del mango para fomentar su consumo, principalmente de las variedades de interés comercial (CONASPROMANGO, 2012), mientras que las variedades comercializadas a nivel local carecen de estudios que impulsen la promoción de su consumo por el aporte nutricio-funcional y que permitan agregar valor a la cadena productiva (Sumaya-Martínez et al., 2012). Uno de los compuestos que pueden dar un valor adicional a este fruto son los antioxidantes naturales, los cuales están ampliamente distribuidos en los alimentos vegetales frescos y sus productos, entre ellos la vitamina $E$, vitamina C, carotenoides y compuestos fenólicos, específicamente flavonoides (Ercisli et al., 2008).

De acuerdo con estudios en algunos vegetales (Corrales-Bernal et al., 2014; Kuskoski et al., 2005), frutos como mango y guayaba (Psidium guajava L.) poseen propiedades medicinales o nutracéuticas, las cuales son atribuidas principalmente al conjunto de compuestos fenólicos contenidos, carotenoides totales y a su capacidad antioxidante. Las variedades cultivadas (cultivares) difieren en su contenido de componentes antioxidantes debido a variaciones genotípicas, factores de manejo pre-cosecha, condiciones edafo-climáticas, prácticas agrícolas y etapa de maduración (Maldonado-Astudillo et al., 2014).

Por lo anterior, es de interés conocer la composición de variedades de mango cultivadas en el estado de Guerrero, el principal estado mexicano productor de mango y en la presente investigación se tiene como objetivo determinar las características físicas, químicas y capacidad antioxidante de las variedades de mango Ataulfo, Manila, Irwin y criollo.

\section{MATERIALES Y MÉTODOS}

\section{Material biológico}

Se cosecharon aleatoriamente 40 frutos frescos y sanos de cuatro variedades de mango: Ataulfo, Manila, Irwin y Criollo en una huerta comercial en el municipio de Atoyac de Álvarez, Guerrero, México durante el periodo enero a julio de 2015. Los frutos se cosecharon en dos fases de madurez: fisiológica 'M1' y de consumo 'M2'. Los criterios de madurez se tomaron de la norma mexicana NMX-FF058-SCFI-2006 (Secretaria de Economía, 2006). Los frutos frescos se lavaron con agua clorada ( $5 \% \mathrm{v} / \mathrm{v}$, conteniendo
500 ppm de $\mathrm{NaClO}$ ), se secaron sobre papel absorbente, se separaron por estado de madurez y cultivar, y se almacenaron a $20^{\circ} \mathrm{C}$.

\section{Análisis físicos}

Se utilizó un muestreo no destructivo con 10 frutos frescos como unidad experimental para evaluar el color de epicarpio y mesocarpio, peso, diámetro longitudinal y transversal. El color de la epidermis se midió con un espectrofotómetro (X-rite ${ }^{\circledR}$ mod. Ci62, USA) en dos partes opuestas de la sección ecuatorial de cada fruto para obtener los datos de Luminosidad $(L *)$, cromaticidad $(C)$ y matiz (h). El peso se determinó con una balanza digital (OHAUS ${ }^{\circ}$, USA) con una sensibilidad de $0.1 \mathrm{~g}$, mientras que los diámetros, polar (DT) y longitudinal (DL), fueron medidos con un vernier (Mitutoyo®, Japón) con una sensibilidad de $0.01 \mathrm{~mm}$. Para la determinación de firmeza se utilizó un muestreo destructivo con 10 frutos como unidad experimental. La firmeza se determinó en apego a la NMXFF-058-SCFI-2006 (Secretaria de Economía, 2006) con un penetrómetro digital (TPM modelo GY4, China) con émbolo de $11 \mathrm{~mm}$ de diámetro y un intervalo de presión de 1.75 a $60 \mathrm{~kg} \mathrm{f}$, con el que se registró la fuerza (kg f) requerida para penetrar el fruto.

\section{Análisis químicos}

Se utilizó un muestreo destructivo con 5 frutos frescos como unidad experimental para evaluar los sólidos solubles totales (SST), pH y acidez total titulable (ATT), que se determinaron según lo descrito por Alia-Tejacal et al. (2012). Para realizar estas determinaciones, se homogenizaron $5 \mathrm{~g}$ de pulpa fresca y se ajustaron a un volumen final de $100 \mathrm{~mL}$ con agua destilada. La muestra homogenizada fue utilizada para las mediciones de SST, pH y ATT. La humedad se realizó por el método 20.013 de la AOAC (2005) para frutas ricas en azúcar, el cual consistió en colocar $5 \mathrm{~g}$ de pulpa fresca en estufa a $60^{\circ} \mathrm{C}$ durante $12 \mathrm{~h}$ y posteriormente estimar la pérdida de peso debido principalmente a la volatilización del agua. Las cenizas fueron estimadas por el método 940.26 de la AOAC (2005).

La muestra utilizada para humedad fue incinerada a 600 ${ }^{\circ} \mathrm{C}$, y las cenizas obtenidas correspondieron a los minerales contenidos en la muestra. Los azúcares totales fueron determinados por el método de antrona (Yemm y Willis, 1954). La preparación de la muestra consistió en pesar 0.5 $\mathrm{g}$ de pulpa fresca y colocarla en $50 \mathrm{~mL}$ de agua caliente, homogenizarla y posteriormente filtrarla. Se tomó $0.1 \mathrm{~mL}$ de la solución muestra y se colocó en un tubo de reacción con solución de antrona y posteriormente se midió la absorbancia a $630 \mathrm{~nm}$ con un espectrofotómetro (Thermo Scientific Evolution 200, Estado Unidos). La concentración 
se calculó mediante una curva patrón de glucosa.

\section{Análisis de compuestos antioxidantes}

Pulpa fresca de mango (100 g) fue secada en estufa (35 a $40{ }^{\circ} \mathrm{C}$ ) durante $72 \mathrm{~h}$; posteriormente se molió en un molino para café (Molino Krups ${ }^{\circledR}$ Acero Inoxidable GX4, Alemania). Para la preparación de los extractos se pesó $1 \mathrm{~g}$ de muestra seca molida, se maceró con $25 \mathrm{~mL}$ de alcohol (metanol o etanol) con agitación orbital a 100 rpm durante $24 \mathrm{~h}$ en oscuridad. Los extractos se aforaron a $50 \mathrm{~mL}$ y se concentraron a presión reducida en un rotaevaporador (Dragon ${ }^{\circledR}$, RE 100, China) hasta un volumen final de $5 \mathrm{~mL}$.

A partir de los extractos se determinó el contenido de fenoles totales por el método de Folin-Ciocalteu (Waterman y Mole, 1994) con una curva patrón de ácido gálico (SigmaAldrich ${ }^{\circledR}$ ). La determinación de flavonoides se hizo con el método colorimétrico de $\mathrm{AlCl}_{3}$ (Chang et al., 2002) con una curva estándar de catequina (Sigma-Aldrich $®$ ) y la actividad atrapadora de radicales libres se realizó con el método del radical libre DPPH (2,2-difenil-1-picrilhidracilo) descrito por Liu y Yao (2007). La determinación de vitamina C fue realizada con el método espectrofotométrico de la derivada de segundo orden. Para ello $1 \mathrm{~g}$ de pulpa de mango homogenizado fresco fue llevado a un volumen de $10 \mathrm{~mL}$ de agua destilada. Se colocaron $0.2 \mathrm{~mL}$ de esta solución en un tubo y se agregaron $0.8 \mathrm{~mL}$ de TCA a $10 \%$. La mezcla se agitó vigorosamente y se colocó en baño de hielo por 5 min. Posteriormente se centrifugó a 3000 rpm durante 5 min, luego el sobrenadante se decantó. Se utilizaron alícuotas de $0.5 \mathrm{~mL}$ de sobrenadante para la estimación de ácido ascórbico y se agregaron $200 \mu \mathrm{L}$ de reactivo folin y se agitó vigorosamente. Después de un reposo de 10 min se midió su absorbancia a $760 \mathrm{~nm}$ con un espectrofotómetro (Thermo Scientific Evolution 200, Estado Unidos). El contenido de vitamina $\mathrm{C}$ se estimó mediante una curva estándar de ácido ascórbico.

\section{Análisis estadístico}

El experimento se condujo con un arreglo bifactorial (características físicas y químicas) y trifactorial (características funcionales). Los factores evaluados fueron: el cultivar (C) con cuatro niveles y el estado de madurez (M) con dos niveles. Para las características funcionales, el análisis se extendió al factor solución extractora con tres niveles. Se aplicó un análisis de varianza (ANOVA) y una prueba de comparación de medias de Tukey $(P \leq 0.05)$ con el paquete estadístico SAS ver. 9 (SAS Institute, 2009).

\section{RESULTADOS Y DISCUSIÓN}

La biomasa de los frutos evaluados varió entre 150 y 390 g, donde el mayor valor correspondió a la var. Ataulfo (Cuadro 1). Ballinas et al. (2013) reportaron valores entre 180 y $280 \mathrm{~g}$ en Ataulfo cultivado en Chiapas, México, intervalo que fue superado por los frutos evaluados en el presente trabajo. En la norma del CODEX 184-1993 Enmienda 2005 (FAO, 1993) se clasifica a los mangos en función de su masa en las categorías: A (200 a 350 g), B (351 a 550) y C (551 a 800 g). Con este criterio, los frutos de Ataulfo, Manila e Irwin aquí evaluados presentaron calibre ' $A$ '.

Si se utiliza la NOM-188-SCFI-2012, que incluye las especificaciones que debe cumplir el mango Ataulfo, esta variedad analizada se ubica en la categoría extra grande (324 a 606 g), y en categoría de calibre 12 (341 a 379 g). La variedad Criollo no se comercializa en el mercado internacional; no obstante, es un recurso muy abundante en el mercado local y nacional que tiene una producción constante durante casi todo el año, muy buena aceptación e importancia económica en la región (CONASPROMANGO, 2012).

Los frutos de la variedad Irwin mostraron la mayor firmeza entre los frutos evaluados (Cuadro 1). En la NMXFF-058-SCFI-2006 los valores mínimos de firmeza para Ataulfo y Manila es de $15.6 \mathrm{~kg}$ f, por lo que todas las variedades analizadas en el presente estudio cumplen con este criterio. Los frutos en madurez fisiológica presentaron mayor firmeza, para luego disminuir en etapas posteriores; tal disminución se atribuye a la degradación enzimática de polisacáridos como almidón, pectinas, celulosa y hemicelulosa en la etapa de maduración, pues son componentes estructurales de la pared celular (Cárdenas-Coronel et al., 2012; Muy-Rangel et al., 2009). Los frutos de las variedades Ataulfo, Manila e Irwin presentaron mayores valores de firmeza que los reportados por Cárdenas-Coronel et al. (2012), quienes encontraron valores entre 18.46 y $1.22 \mathrm{~kg} \mathrm{f}$.

La variedad con mayor luminosidad fue Ataulfo; las variedades Ataulfo, Manila y Criollo presentaron tonalidades tendientes al amarillo (matiz > 40), mientras que el cv. Irwin presentó tono tendiente al rojo (matiz < 40). De acuerdo con Maldonado-Astudillo et al. (2014), los cambios en la coloración externa de los frutos son característicos del proceso de maduración y son los principales atributos en la selección del fruto durante la cosecha y algunos procesos de comercialización. La luminosidad en frutos es mayor en el estado maduro, lo cual se atribuye a la degradación de clorofilas y a la síntesis de carotenoides durante la maduración. Este parámetro es útil como un indicador de la madurez del fruto (Ballinas-Díaz et al., 2013; PalafoxCarlos et al., 2012).

Los frutos de Ataulfo presentaron mayor acidez total titulable, ATT (Cuadro 2). Estos valores son menores a los 
Cuadro 1. Características físicas de cuatro variedades de mango en dos etapas madurez en Guerrero, México.

\begin{tabular}{|c|c|c|c|c|c|c|c|}
\hline \multirow{2}{*}{ Factor } & \multirow{2}{*}{$\mathrm{DL}(\mathrm{mm})$} & \multirow{2}{*}{$\mathrm{DT}(\mathrm{mm})$} & \multirow{2}{*}{ Peso (g) } & \multirow{2}{*}{ Firmeza (kg f) } & \multicolumn{3}{|c|}{ Color } \\
\hline & & & & & Luminosidad & Croma & Matiz ( $\left.{ }^{\circ} \mathrm{h}\right)$ \\
\hline \multicolumn{8}{|c|}{ Variedad (V) } \\
\hline Ataulfo & $118.8 b$ & $80.3 a$ & 387.8 a & $21 \mathrm{~b}$ & $64.2 \mathrm{a}$ & 45.9 a & $88.7 \mathrm{a}$ \\
\hline Manila & $203.9 \mathrm{a}$ & $63.4 b$ & $229.6 b$ & $25 b$ & $59.2 a b$ & $43.4 \mathrm{a}$ & $89.0 \mathrm{a}$ \\
\hline Criollo & $55.5 d$ & $37.4 \mathrm{~d}$ & $153.8 \mathrm{c}$ & $18 b$ & $56.9 b$ & $44.7 \mathrm{a}$ & $90.5 \mathrm{a}$ \\
\hline Irwin & $70.3 c$ & $54.0 \mathrm{c}$ & $297.0 \mathrm{~b}$ & $37 \mathrm{a}$ & $37.3 \mathrm{c}$ & $31.5 b$ & $53.0 \mathrm{~b}$ \\
\hline DSH (0.05) & 167.3 & 7.2 & 102.6 & 0.9 & 5.9 & 4.2 & 6.1 \\
\hline \multicolumn{8}{|c|}{ Estado de madurez (M) } \\
\hline M1 & $124.4 \mathrm{a}$ & $70.5 \mathrm{a}$ & $280.1 \mathrm{a}$ & $47.8 \mathrm{a}$ & $55.9 b$ & $40.3 b$ & $100.2 \mathrm{a}$ \\
\hline M2 & 121.8 a & $68.3 \mathrm{a}$ & 278.9 a & $38.8 b$ & $59.9 a$ & $46.8 \mathrm{a}$ & $65.8 b$ \\
\hline DSH (0.05) & 82.9 & 3.5 & 50.8 & 0.5 & 2.9 & 2.1 & 3.0 \\
\hline CV & 122.2 & 9.9 & 31.9 & 22.5 & 8.7 & 8.3 & 6.2 \\
\hline$V \times M$ & ns & $\star *$ & $* *$ & ** & $\star *$ & $\star *$ & $\star *$ \\
\hline
\end{tabular}

DL: diámetro longitudinal; DT: diámetro transversal; IF: índice de forma (DL/DT). M1: madurez fisiológica; M2: madurez de consumo. Medias con letras iguales en cada columna no son estadísticamente diferentes en cada factor (Tukey, 0.05); DSH: diferencia significativa honesta; CV: coeficiente de variación. ns: no significativo, * significancia con $\mathrm{P} \leq 0.05 ; * *$ significancia con $\mathrm{P} \leq 0.01$.

reportados por Osuna et al. (2002) en frutos de mango Ataulfo del estado de Nayarit, México. Al día uno de almacenamiento los frutos presentaban valores de acidez de $3 \%$ de ácido cítrico; en cambio, a madurez de consumo (10 días de almacenamiento) los valores de acidez fueron de 0.3 a $0.4 \%$ de ácido cítrico. Esta tendencia también fue encontrada en este estudio, ya que los frutos en madurez fisiológica presentaron los valores mayores de ATT.

Los ácidos orgánicos (cítricos, ascórbico y málico) son importantes durante la maduración de la fruta, debido a que pueden ser una fuente de energía durante la respiración y por ello su contenido tiende a decrecer con el desarrollo (Sampaio et al., 2007). Paralelo a la pérdida de acidez, hay un incremento significativo del $\mathrm{pH}$ en los frutos durante el proceso de maduración (Maldonado-Astudillo et al., 2014). Los frutos en estado maduro presentaron $\mathrm{pH}$ mayor que los frutos en madurez fisiológica, con valores similares a los reportados por Palafox-Carlos et al. (2012).

No se detectaron diferencias significativas en el contenido de sólidos solubles totales (SST) entre las variedades (Cuadro 2). Valores similares a los aquí encontrados (11 $\pm 4 \%$ SST) fueron reportados por Casierra-Posada y Guzmán (2009) para la variedad Irwin. Los frutos maduros presentaron valores superiores en SST debido a la acumulación de azúcares libres durante la maduración, producto de la hidrólisis del almidón por la acción de las amilasas (Cárdenas-Coronel et al., 2012).

No se observaron diferencias significativas en los con- tenidos de humedad y cenizas entre las variedades estudiadas, pero si entre estados de madurez, ya que los frutos en madurez fisiológica presentaron mayor contenido de humedad que en estados posteriores, como lo reportaron anteriormente Briceño et al. (2005) y Mejía et al. (2007). El contenido de humedad de los frutos está fuertemente influenciado por factores como la etapa de maduración, transpiración, lesiones, condiciones de almacenamiento (temperatura, humedad relativa, tiempo de almacenamiento), entre otros factores (Maldonado-Astudillo et al., 2014).

La variedad Irwin presentó el mayor contenido de AT, seguida de las vars. Ataulfo y Manila. Stafford (1983) reportó un contenido de AT en frutos de mango de $16.8 \mathrm{~g}$, similar a los encontrados en la etapa de madurez fisiológica en variedades aquí estudiadas (Cuadro 2). En madurez de consumo los frutos presentaron valores más altos. El contenido de AT en los frutos está influenciado por factores como la variedad, estado de madurez, contenido de agua, lesiones, entre otros (Maldonado-Astudillo et al., 2014).

De acuerdo con Soto et al. (2004), la acidez varía con la maduración de la fruta, ya que el pH se incrementa y el contenido de vitamina $C$ disminuye a medida que el contenido de azúcares y carotenos aumentan marcadamente. El contenido de vitamina $\mathrm{C}$ fue significativamente mayor en las vars. Manila y Criollo en madurez de consumo. Los valores encontrados en las cuatro variedades analizadas son superiores a los reportados por CorralesBernal et al. (2014) en frutos de mango cosechados en Colombia (0.09 a $1.86 \mathrm{mg} \mathrm{g}^{-1}$ ). Según Stafford (1983), el 
Cuadro 2. Características químicas en base fresca de cuatro variedades de mango cosechados en dos etapas de madurez en Guerrero, México.

\begin{tabular}{|c|c|c|c|c|c|c|c|c|}
\hline Factor & ATT $(\%)$ & $\mathrm{pH}$ & SST ('Brix) & IS SST/ATT & Humedad (\%) & Cenizas (\%) & AT $(\%)$ & Vit C $\left(\mathrm{mg} \mathrm{g}^{-1}\right)$ \\
\hline \multicolumn{9}{|c|}{ Variedad (V) } \\
\hline Ataulfo & $0.3 \mathrm{a}$ & $1.6 b$ & 9.5 & 31.6 & 82.4 & 0.5 & $27.1 \mathrm{~b}$ & $17.7 b$ \\
\hline Manila & $0.2 b$ & $3.5 \mathrm{a}$ & 11.3 & 56.5 & 69.3 & 0.4 & $21.7 b$ & $48.1 \mathrm{a}$ \\
\hline Criollo & $0.1 \mathrm{c}$ & $3.9 \mathrm{a}$ & 10.7 & 107 & 60.3 & 0.4 & $16.4 \mathrm{c}$ & $34.7 \mathrm{a}$ \\
\hline Irwin & $0.1 \mathrm{c}$ & $3.8 \mathrm{a}$ & 8.5 & 85 & 68.0 & 0.4 & $31.0 \mathrm{a}$ & $18.7 b$ \\
\hline DSH (0.05) & 0.04 & 0.5 & 3.0 & 46.1 & 53.3 & 0.1 & 4.9 & 38.3 \\
\hline \multicolumn{9}{|c|}{ Estado de madurez $(\mathrm{M})$} \\
\hline M1 & $0.2 \mathrm{a}$ & $2.5 b$ & $8.9 b$ & $44.5 b$ & $83.6 \mathrm{a}$ & 0.4 & $19.4 b$ & $36.3 \mathrm{a}$ \\
\hline M2 & $0.1 b$ & $3.7 \mathrm{a}$ & $11.0 \mathrm{a}$ & $110 a$ & $59.7 b$ & 0.5 & $27.5 \mathrm{a}$ & $24.3 b$ \\
\hline DSH (0.05) & 0.02 & 0.3 & 1.5 & 24.1 & 28.1 & 0.1 & 2.6 & 19.4 \\
\hline$C V$ & 16.4 & 24.0 & 14.4 & 24.4 & 2.5 & 19.5 & 23.8 & 8.5 \\
\hline$V \times M$ & $\star \star$ & $\star \star$ & ** & $\star *$ & * & * & $\star \star$ & $\star *$ \\
\hline
\end{tabular}

ATT: acidez total titulable; SST: sólidos solubles totales; IS: índice de sabor (SST/ATT); AT: azúcares totales; VIT C: vitamina C; M1: madurez fisiológica; M2: madurez de consumo. Medias con letras iguales en cada columna no son estadísticamente diferentes en cada factor (Tukey, 0.05); DSH: diferencia significativa honesta; CV: coeficiente de variación. * Significancia con $\mathrm{P} \leq 0.05$; ** significancia con $\mathrm{P} \leq 0.01$.

contenido de vitamina $\mathrm{C}$ depende de la variedad de mango y el estado de madurez. La vitamina C es la más lábil en los alimentos, por lo que se utiliza con un índice de calidad en los productos procesados.

Li et al. (2014) y Corrales-Bernal et al. (2014) encontraron que los frutos de mango contienen componentes nutrimentales importantes como el ácido ascórbico, a-tocoferol, carotenoides, flavonoides, polifenoles, así como una considerable capacidad antioxidante, los cuales varían entre cultivares, estados de madurez y condiciones edafo-climáticas. Estos autores concluyeron que algunas variedades criollas poseen un contenido importante en fenoles totales y carotenoides, especialmente en la pulpa madura, que le confieren propiedad antioxidante comparable; en algunos casos los contenidos son aún mayores a los de otras variedades de mango más estudiadas y con mayor valor comercial.

El contenido de compuestos fenólicos en extracto metanólico fue significativamente mayor en las vars. Irwin y Ataulfo, seguidos de Manila y Criollo (Cuadro 3). Estos valores son superiores a los reportados por Robles-Sánchez et al. (2013) en mango Ataulfo con un contenido de

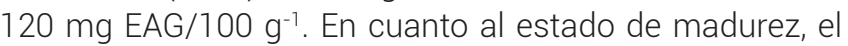
mayor contenido de polifenoles se encontró en frutos con madurez fisiológica. De acuerdo con Robles-Sánchez et al. (2013), los fenoles totales tienden a disminuir con la maduración y se asocian a con la senescencia del fruto.

Con respecto a los flavonoides, el mayor contenido se encontró en la variedad Manila y en extracto etanólico (438.4 mg CAT/100 g), seguida de Ataulfo en ambos extractos alcohólicos. En un estudio de Kuskoski et al. (2005) la pulpa de mango presentó una mayor actividad antioxidante y una mayor concentración de compuestos fenólicos totales comparada con la pulpa de frutos como uva (Vitis vinifera), guayaba (Psidium guajava) y piña (Ananas comosus).

La variedad Ataulfo presentó la mayor actividad atrapadora de radicales libres en extracto metanólico (22.45 \% inhibición DPPH), Manila en acuoso (34.2\%), Criollo no mostró diferencias entre los 3 extractos utilizados (24 a $28 \%$ ) e Irwin en acuoso (30.2\%). Las cuatro variedades de mango analizadas mostraron el mayor porcentaje de inhibición en madurez fisiológica (M1). Resultados similares fueron reportados por Palafox-Carlos et al. (2012), quienes concluyeron que el estado de madurez de frutos de mango Ataulfo influye en el contenido de compuestos fenólicos y flavonoides, lo cual está relacionado con la capacidad antioxidante.

Palomo et al. (2009) reportaron valores superiores de actividad antioxidante (expresada como porcentaje de decoloración de la solución de DPPH) en extractos metanólicos de frutas de ciruela, Prunus domestica (71 \%), manzana Golden, Malus pumila (73 \%), uva Red Globe, Vitis vinifera (60\%) y sandia, Citrullus vulgaris (74\%); en hortalizas, los mayores valores fueron para acelga, Beta vulgaris (58 \%) y pepino dulce, Solanum muricatum (80 \%). Por su parte Robles-Sánchez et al. (2013) reportaron que el consumo de mango puede proveer cantidades significativas de 


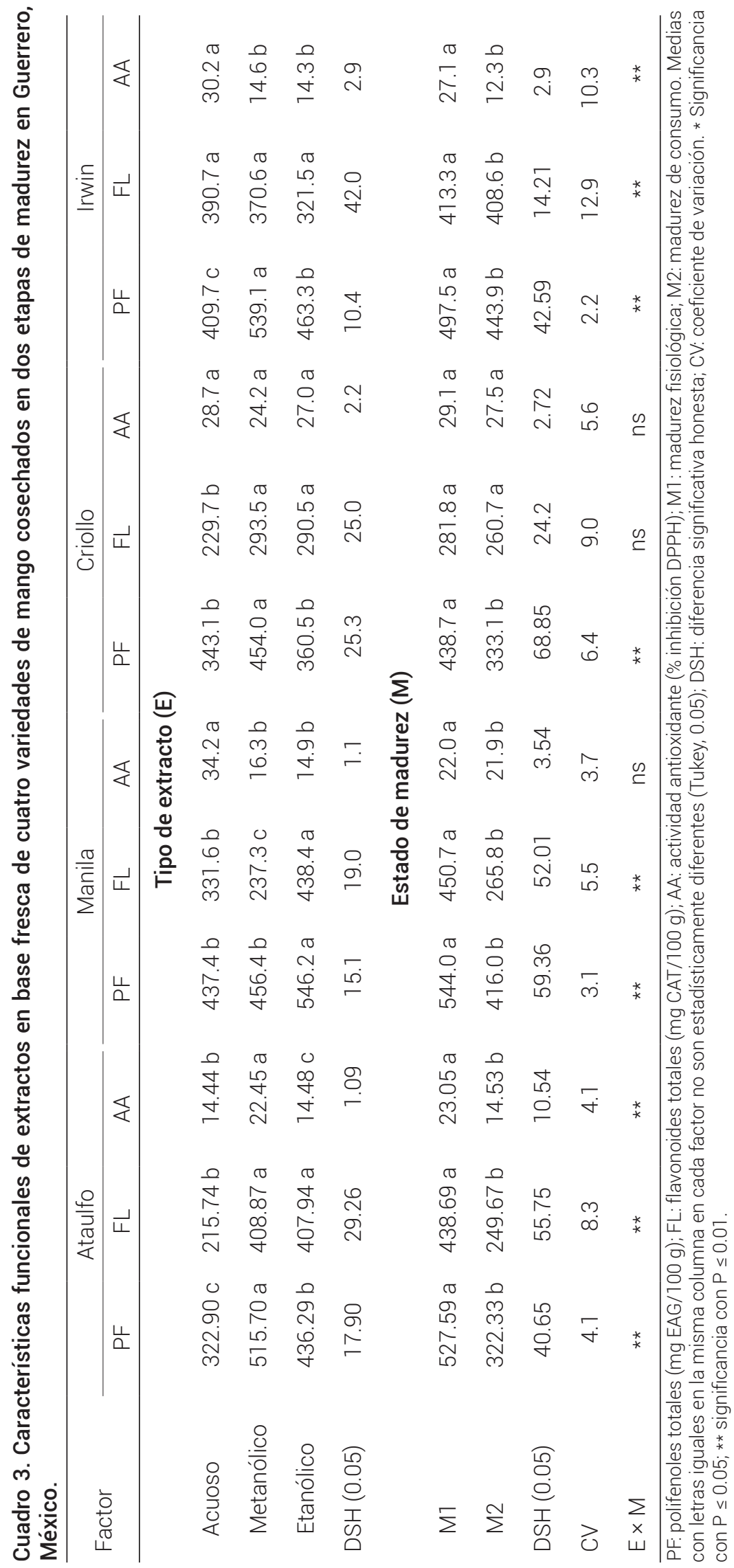


compuestos bioactivos con actividad antioxidante; sin embargo, es necesario desarrollar técnicas que disminuyan el deterioro del fruto después del procesamiento mínimo.

\section{CONCLUSIONES}

Las características físicas y químicas de las variedades Ataulfo, Manila, Criollo e Irwin cultivados en Guerrero, México satisfacen los requerimientos de la Norma NMX-FF058-SCFI-2006 de calidad de mango fresco para comercio nacional, y la variedad Ataulfo cumple con la categoría extra grande de la NOM-188-SCFI-2012. La alta actividad atrapadora de radicales libres de las variedades Manila, Irwin y Criollo les confieren importancia funcional a sus frutos.

\section{AGRADECIMIENTOS}

Se agradece el apoyo económico otorgado al proyecto FOMIX 249611 (CONACYT) y al proyecto semilla2014 (UAGro) por las becas otorgadas, así como a Verónica Flores Casamayor de CINVESTAV Querétaro por la asistencia técnica brindada.

\section{BIBLIOGRAFÍA}

Alia-Tejacal I., Y. I. Astudillo-Maldonado-, C. A. Núñez-Colín, L. A. ValdezAguilar, S. Bautista-Baños, E. García-Vázquez, R. Ariza-Flores y F. Rivera-Cabrera (2012) Caracterización de frutos de ciruela mexicana (Spondias purpurea L.) del sur de México. Revista Fitotecnia Mexicana 35 (Núm. Esp. 5):21-26.

AOAC, Association of Official Analytical Chemists International (2005) Official Methods of Analysis of Association of Official Analytical Chemists International. $18^{\text {th }}$ edition. Association of Official Analytical Chemists International. Maryland, USA. pp:27-30.

Ballinas, D. E. J., G. Vela, G., E. J. López, Z., O. A. Aguilar, N., A. Caballero, R., P. I. Meza, G., A. Pérez, J., L. E. Flores, G. y J. M. León, G (2013) Mango: Cultivo, Tratamiento Pre y Postcosecha. Propiedades Nutrimentales y Funcionales. Editorial UNICACH. Universidad de Ciencias y artes de Chiapas. Tuxtla Gutiérrez, Chiapas, México. $73 \mathrm{p}$

Briceño S., J. Zambrano, W. Materano, I. Quintero y A. Valera (2005) Calidad de los frutos de mango 'Bocado' madurados en la planta y fuera de la planta cosechados en madurez fisiológica. Agronomía Tropical 55:461-473

Cárdenas-Coronel W. G., R. Vélez-dela Rocha, J. H. Siller-Cepeda, T. OsunaEnciso, M. D. Muy-Rangel y J. A Sañudo-Barajas (2012) Cambios en la composición de almidón, pectinas y hemicelulosa durante la maduración de mango (Mangifera indica cv. 'Kent'). Revista Chapingo Serie Horticultura 18:5-19.

Casierra-Posada F. y J. A. Guzmán (2009) Efecto del portainjerto y del injerto intermedio sobre la calidad de fruta en mango (Mangifera indica L.). Agronomía Colombiana 27: 367-374.

Chang C. C., M. H. Yang, H. W. Wen y J. C. Chern (2002) Estimation of total flavonoids content in propolis by two complementary colorimetric methods. Journal of Food and Drug Analysis 10: 178182.

CONASPROMANGO, Consejo Nacional del Sistema Producto Mango (2012) Plan Rector Nacional de Sistema Producto Mango. Comité Nacional Sistema Producto Mango. Tecomán, Colima, México. 57 p.

Corrales-Bernal A., M. E. Maldonado, L. A. Urango, M. C. Franco y B. A. Rojano (2014) Mango de azúcar (Mangifera indica), variedad de Colombia: características antioxidantes, nutricionales y sensoriales.
Revista Chilena de Nutrición 41:312-318.

Ercisli S., M. Akbulut, M. Ozdemir, M. Sengul and E. Orhan (2008) Phenolic and antioxidant diversity among persimmon (Diospyrus kaki L.) genotypes in Turkey. International Food Sciences and Nutrition 59:477-482

FAO, Organización de las Naciones Unidas para la Alimentación y la Agricultura (1993) CODEX Norma del codex para el mango (CODEX STAN 184-1993.). Enmienda 2005. Normas Internacionales de Alimentos. www.codexalimentarius.org/input/download/standards/315/CXS_184s.pdf (Junio 2016).

FAOSTAT (2012) Importaciones y exportaciones/país por producto/ mango (Bases de Datos Estadístico Sustantivo de la FAO). http://faostat3.fao.org/home/S (Junio 2016).

Huang S. and K. Huang (2007) Increased U.S. imports of fresh fruit and vegetables. Report from the Economic Research Service. USDA-ERS. http://www.ers.usda.gov/ media/187841/ fts32801_1_.pdf (Junio 2016).

Kuskoski E. M., A. G. Asuero, A. M. Troncoso, J. Mancini-Filho y R. Fett (2005) Aplicación de diversos métodos químicos para determinar actividad antioxidante en pulpa de frutos. Food Science and Technology (Campinas) 25:726-732.

Li L., S. Wang, J. Chen, J. Xie, H. Wu, R. Zhan and W. Li (2014) Major antioxidants and in vitro antioxidant capacity of eleven mango (Mangifera Indica L.) cultivars. International Journal of Food Properties 17:1872-1887.

Liu Q. and H. Yao (2007) Antioxidant activities of barely seed extracts. Food Chemistry 102:732-737.

Maldonado-Astudillo Y. I., I. Alia-Tejacal, C. A. Núñez-Colín, J. JiménezHernández, C. Pelayo-Zaldívar, V. López-Martínez and S. ValleGuadarrama (2014) Postharvest physiology and technology of Spondias purpurea L. and S. mombin L. Scientia Horticulturae 174:193-206

Mejía G., L. F., H. A. Martínez C., J. E. Betancourt G. y C. E. Castrillón C. (2007) Aprovechamiento del residuo agroindustrial del mango común (Mangifera indica L.) en la obtención de azúcares fermentables. Ingeniería y Ciencia 3:41-62. http://www.ers.usda.gov/Publications/fts/2007/08Aug/fts32801/fts32801.pdf. (Septiembre 2008).

Muy R. D., B. Espinoza V., J. Siller C., J. A. Sañudo B., B. Valdez T. y T. Osuna E (2009) Efecto del 1-metilciclopropeno (1-MCP) y de una película comestible sobre la actividad enzimática y calidad poscosecha de mango 'Ataulfo'. Revista Fitotecnia Mexicana 32:53-60.

Osuna G., J. A., M. L. Guzmán R., B. Tovar G., M. Mata M. O. y V. A. Vidal M. (2002) Calidad del mango 'Ataulfo' producido en Nayarit, México. Revista Fitotecnia Mexicana 25:367-374.

Palafox-Carlos H., E. Yahia, M. A. Islas-Osuna, P. Gutierrez-Martinez, M. Robles-Sánchez and G. A. González-Aguilar (2012) Effect of ripeness stage of mango fruit (Mangifera indica L., CV. Ataulfo) on physiological parameters and antioxidant activity. Scientia Horticulturae 135: 7-13.

Palomo G., I., M. Gutiérrez C., L. Astudillo S., C. Rivera S., C. Torres U., L. Guzmán J., R. Moore-Carrasco, G. Carrasco S. y M. Alarcón L. (2009) Efecto antioxidante de frutas y hortalizas de la zona central de Chile. Revista Chilena de Nutrición 36:152-158.

Robles-Sánchez R. M., M. A. Rojas-Graü, I. Odriozola-Serrano, G. GonzálezAguilar and 0. Martin-Belloso (2013) Influence of alginate-based edible coating as carrier of antibrowning agents on bioactive compounds and antioxidant activity in fresh-cut Kent mangoes. LWT-Food Science and Technology 50:240-246.

SAGARPA, Secretaría de Agricultura, Ganadería, Desarrollo Rural, Pesca y Alimentación (2014) México, el mayor exportador de mango. Secretaría de Agricultura, Ganadería, Desarrollo Rural, Pesca y Alimentación. México. http://www.sagarpa.gob.mx/saladeprensa/2012/Paginas/2014B289.aspx (Junio 2016).

Sampaio S. A., P. S. Bora, H. J. Holschuh and S. M. Silva (2007) Postharvest respiratory activity and changes in some chemical constituents during maturation of yellow mombin (Spondias mombin L.) fruit. Ciencia e Tecnologia de Alimentos 27:511-515.

SAS Institute (2009) SAS paquete estadístico ver. 9.0

Secretaria de Economía (2006) NMX-FF-058-SCFI-2006. Productos alimenticios no industrializados para consumo humano-fruta fresca-mango (Mangifera indica L.)-especificaciones. Secretaria de Economía. México. D.F. 20 p. 
Secretaria de Economía (2012) NOM-188-SCFI-2012. Norma Oficial Mexicana. Mango Ataulfo del Soconusco, Chiapas. (Mangifera caesia Jack ex Wall). Especificaciones y métodos de prueba. Diario oficial de la Federación. México. D.F. 29 de noviembre de 2012

Soto E., L. Avilán, E. Unai, M. Rodríguez y J. Ruiz (2004) Comportamiento y características de algunos cultivares promisorios de mango. Agronomía Tropical 54:179-201.

Stafford A. E (1983) Mango. In: Handbook of Tropical Foods. Chan H. T. (ed.). Marcel Dekker Inc. New York. pp:399-432.
Sumaya-Martínez M. T., L. M. Sánchez H., G. Torres G. y D. García P. (2012) Red de valor del mango y sus desechos con base en las propiedades nutricionales y funcionales. Revista Mexicana de Agronegocios 30:826-833

Waterman P.G. and S. Mole (1994) Analysis of Phenolic Plant Metabolites. Methods in Ecology. Blackwell Scientific Publications. Boston. $238 \mathrm{p}$.

Yemm E. W and A. J. Willis (1954) The estimation of carbohydrates in plant extracts by anthrone. Biochemistry Journal 57:508-514. 